To look at the NT first as a sea of manuscripts leads one to reconsider the »textuality « of the NT. Digital editions for various Humanities texts or works have a »decanonizing effect, « as argued by S. Mombert (Mombert). But as long as readers ask to read the New Testament, its identity as a coherent and complete text will probably endure, even in a very new format, like the movie published at the end of 2019 by KoineGreek.com that uses the Greek Gospel according to Mark as script.

\title{
Nichts Neues für die Alte Kirchengeschichte? Das Internet als Ernstfall bistorischer Heuristik
}

Katharina Heyden

Bibliothek der Kirchenväter online, Université de Fribourg, online: https://bkv.unifr.ch/ (07.06.2020). - Corpus Thomisticum, Universidad de Navarra, online: https://www.corpusthomisticum.org/ (07.06.2020). - e-codicology, Technische Universität Darmstadt, online: http://www. ecodicology.org/ (07.06.2020). - Franz Fischer (Hg.), Magistri Guillielmi Autissiodorensis Summa de officiis ecclesiasticis. Kritisch-digitale Erstausgabe, Cologne Center for eHumanities $\mathrm{CceH}$, online: http://guillelmus.uni-koeln.de/tcrit/tcrit_prologus (07.06.2020). -James J. O’Donnell (Hg.), The »Confessions « of Augustine. Text and Commentary, Oxford University Press Oxford 1992. An Electronic Edition, online: http://www.stoa.org/hippo/index.html (07.06.2020). - Pinakes. Textes et manuscrits grecs, Institut de recherche et d'histoire de textes, online: https://pinakes.irht.curs. fr/ (05.07.2020). - prometheus. Das verteilte digitale Bildarchiv für Forschung und Lehre, https:// www.prometheus-bildarchiv.de/ (07.06.2020). - Wikipedia. Die Freie Enzyklopädie, Art. Confessiones, online: https://de.wikipedia.org/w/index.php?title=Confessiones\&oldid=199339509 (Permalink, letzte eingesehene Version vom 23.03.2020). - Zentrum für Augustinus-Forschung, Julius-Maximilians-Universität Würzburg, online: https://www.augustinus.de/ (07.06.2020).

\section{Einführung: Nichts Neues?}

Die Kirchengeschichte des Mittelalters kann sich rühmen, mit dem Index Thomisticus vor über 70 Jahren den Anstoß für die Entwicklung von Digital Humanities gegeben zu haben. Heute erweist sich das Internet als Ernstfall der historischen Heuristik, also der Kunst, mit begrenztem Wissen in einem unübersichtlichen Feld von Informationen diejenigen Quellen zu finden, die zur Beantwortung einer Forschungsfrage geeignet sind. Das Suchen und Finden geeigneter Quellen ist kein Problem, mit dem erst das Internetzeitalter die historische Forschung konfrontieren würde. Die Heuristik, von J.G. Droysen in seinem »Grundriss der Historik«als »Bergmannskunst« des Historikers bezeichnet ( $\mathbb{2 0}$ : http://www. deutschestextarchiv.de/book/view/droysen_historik_1868?p=22 [07.06.2020]), ist schon immer eine heikle Kunst gewesen. Wie kann man sinnvoll auswählen, 
ohne den Auswahlbereich auch nur annähernd zu überblicken? Und wie lassen sich Erkenntnisse, die aus mehr oder weniger zufällig ans Licht gekommenen Bruchstücken der Vergangenheit gewonnen wurden, angemessen kontextualisieren? Der Mediävist und langjährige Direktor des Deutschen Historischen Instituts in Rom, A. Esch, hat einmal darauf hingewiesen, dass Historiker die Tendenz hätten, wie Kinder beim Pfeilwerfen auf eine weiße Wand nachträglich eine Zielscheibe um ihren Pfeil zu malen und damit zu suggerieren, dass sie mit ihren Erkenntnissen direkt ins Zentrum eines Problems getroffen hätten (A. Esch, Überlieferungschance und Überlieferungszufall als methodisches Problem des Historikers: HZ 240 [1985] 529-570). Dass man die eigenen Funde und Erkenntnisse niemals endgültig in das große Bild der Geschichte einzeichnen kann, gehört zum faszinierenden Geschäft historischer Forschung. Es gibt im Blick auf das Gesamt der Vergangenheit ungleich viel mehr Unbekanntes als Bekanntes. Die Fragmente der Vergangenheit, die wir überblicken können, sind verschwindend klein im Vergleich zu dem, was uns entzogen ist. Die Analogie zum Internet lautet: Die Informationen und Quellen, die wir bei Internetrecherchen tatsächlich auffinden, sind verschwindend klein im Vergleich zu dem, was zwar vorhanden ist, sich aber unserem Überblick und Zugriff entzieht. Das Internet verschärft also die Herausforderungen, vor denen kirchenhistorische Forschung schon immer und ganz grundsätzlich steht. Insofern ist das Internet als Ernstfall (kirchen)historischer Forschung anzusehen.

Damit ist auch das Dilemma benannt, vor dem der mit diesem Beitrag unternommene Versuch steht, einen möglichst repräsentativen Einblick in die Bedeutung von Internet und Digitalisierung für die kirchenhistorische Forschung zu geben. Konnte M. Wallraff in seinen zwischen 1997 und 2005 in fünf Teilen publizierten »Patristischen Arbeitshilfen im Internet« (in: ZAC 1 [1997] 129-130; 3 [1999], 120-122; 4 [2000] 158-160; 6 [2002] 348-352, DOI: https://doi.org/10.1515/ zach.2002.022 sowie 8 [2007] 358-371, DOI: https://doi.org/10.1515/zach.8.2.358 [beide 07.06.2020]) noch einen annähernd vollständigen Überblick geben, so ist dies heute unmöglich geworden. Denn das »Bergwerk « ist seither nicht nur auf unübersichtliche Ausmaße angewachsen, es ändert seine Ressourcen und deren Repräsentation auch so rasant und beständig, dass jeder Bericht darüber nichts anderes als eine Momentaufnahme sein kann. Wenn die Metapher vom DartSpiel berechtigt ist, dann gehört jedoch gerade der Verzicht auf den Anspruch, etwas Repräsentatives gefunden zu haben, zu den Tugenden einer um redliche Methodik bemühten Kirchengeschichte. Aus dieser ernüchternden Erkenntnis folgt zweierlei für die folgende Darstellung: Erstens kann es keinen »Überblick «, sondern allenfalls einen »Einblick « in digitale Hilfsmittel zur QuellenerschlieBung für die antike und mittelalterliche Kirchengeschichte geben. Zweitens wähle ich für eine exemplarische Recherche ein Beispiel, bei dem zumindest die repräsentative und nachhaltige Geltung des Objekts (wenn schon nicht der Recherche) unzweifelhaft sein dürfte. Dass die Confessiones des Augustinus eines der berühmtesten und einflussreichsten Werke des berühmtesten und einflussreichsten 
lateinischen Kirchenvaters sind, kann man wohl ohne Übertreibung behaupten. Was findet und was erfährt man von diesem Klassiker der Kirchengeschichte im April 2020 im Internet? Abschließend werden die in Einblick und Fallbeispiel beobachteten Phänomene im Blick auf die These vom Internet als »Ernstfall« kirchenhistorischer Heuristik reflektiert.

\section{Einblick: Digitale Quellenerschließung}

Im Jahr 1949, lange bevor Begriffe wie Digitalisierung und Computerlinguistik in aller Munde waren, gründete der italienische Jesuitenpater Roberto Busa (1913-2011) mit dem Index Thomisticus (https://www.corpusthomisticum.org/ it/index.age [07.06.2020]) das erste umfangreiche maschinell durchsuchbare Textkorpus, welches seit den 1960er Jahren für die Hypertexterstellung wegbereitend wurde. Busa gilt deshalb vielen - auch ausweislich des wikipedia-Artikels (https://de.wikipedia.org/wiki/Roberto_Busa [07.06.2020]) - als Erfinder der Digital Humanities.

Heute ist der Index eingebunden in das im Jahr 2000 gegründete Corpus Thomisticum. Dass diese Webseite, abgesehen von kurzen Einleitungen in elf modernen Sprachen, gänzlich in Latein gehalten ist, bildet einen reizvollen Kontrapunkt zu der bahnbrechenden technischen Pionierleistung, die mit dem Index Thomisticus verbunden ist und die weit über die Theologie hinaus ihre Wirkung entfaltet hat. Das von der Universität von Navarra betreute Projekt umfasst nach eigenen Angaben fünf Teilaspekte. Es bietet

- vollständigen Zugang zu den 117 Werken des Thomas von Aquin in den vom Redaktionsteam jeweils als beste befundenen gedruckten Editionen,

- eine möglichst vollständige Bibliographie über Forschungsliteratur vom 13. Jahrhundert bis heute,

- einschlägige Lexika und andere Hilfsmittel,

- eine Suchmaschine, die Wörter, Sätze, Zitate und Referenzen auffindet, zusammenstellt und ordnet (= der oben erwähnte Index Thomisticus),

- Digitalisate der wichtigsten mittelalterlichen Manuskripte der Werke von Thomas von Aquin.

Diese Liste kann exemplarisch als Zusammenfassung der wichtigsten Hilfsmittel gelesen werden, die das Internet heute für die kirchengeschichtliche Forschung insgesamt zur Verfügung stellt. Dabei repräsentieren die ersten drei Aspekte die nachträgliche Digitalisierung (häufig im PDF- oder rtf-Format) und zunehmend auch die digitale Aufbereitung von bereits publizierten Printmedien, die zwar den Zugang zu bereits gedruckten Werken enorm erleichtert, aber keinen genuinen Einfluss auf die Entwicklung der Forschungsdiskurse hat. Die letzten beiden Aspekte stehen dagegen für die Entwicklung des Faches im Sinne der »Digital Humanities «, also der wechselseitigen Beeinflussung von technischen Möglichkeiten und Forschungsdiskursen. 


\section{a) Digital aufbereitete Printmedien}

Im Blick auf die nachträgliche Digitalisierung und digitale Aufbereitung von bereits gedruckten Texteditionen sind die wichtigsten, weil umfassendsten Datenbanken für griechische Texte der Thesaurus Linguae Graecae (http://stephanus. tlg.uci.edu/ [07.06.2020]), für lateinische kirchenhistorische Werke die Patrologia Latina Database (http://pld.chadwyck.co.uk/ [07.06.2020]) und für die deutsche Geschichte des Mittelalters die digitalen Monumenta Germaniae Historica (https://www.dmgh.de/ [07.06.2020]). Auch die Bibliotheca Augustana (http:// www.hs-augsburg.de/ harsch/augustana.html [07.06.2020]) erweist sich als Fundgrube für Quellenschriften verschiedenster Sprachen aus dem 7. Jh. v. Chr. bis ins 20. Jh. n.Chr., darunter viele kirchenhistorisch relevante Texte. Während die MGH ausnahmslos textkritische Editionen bieten, muss dies bei den anderen drei genannten Datenbanken jeweils im Einzelfall geprüft werden. Für die konkrete kirchenhistorische Arbeit bedeutet dies, dass die Datenbankrecherche die Konsultation der maßgeblichen Texteditionen nicht in jedem Fall ersetzen kann. Die maschinelle Durchsuchbarkeit der in den Datenbanken erfassten Quellentexte bereichert die kirchenhistorische Arbeit jedoch enorm. Sie erleichtert textund corpusübergreifende Analysen zu Textvarianten, Begriffen und sprachlichen Motiven. So gewinnen mit den technischen Möglichkeiten überlieferungs- und rezeptionsgeschichtliche Fragestellungen und Forschungen zu Intertextualität gegenüber der sorgfältigen und detaillierten Interpretation einzelner Werke oder Passagen nach dem Ideal des »close reading « an Bedeutung.

\section{b) Digitalisierte Handschriften}

Neben der Erschließung von bereits gedruckten Texten ist die Digitalisierung vor allem für die Erforschung mittelalterlicher Manuskripte von unschätzbarer Bedeutung.

Die umfassendste und am solidesten aufbereitete Handschriftendatenbank ist »Pinakes « https:// pinakes.irht.cnrs.fr/ (07.06.2020). 1971 in Toronto gegründet, seit 1993 vom Pariser Institut de recherche et d'histoire des textes betrieben und seit 2008 online, bietet diese Datenbank Katalogeinträge und Forschungsliteratur zu griechischen Handschriften bis zum 16. Jh. sowie nach Möglichkeit auch die Links zu den Digitalisaten der Manuskripte aus Bibliotheken der ganzen Welt. Für die Bestände der Schweiz hat sich das in Fribourg ansässige Projekt e-codices https://www.e-codices.unifr.ch/de (07.06.2020) zum Ziel gesetzt, sämtliche mittelalterlichen Handschriften in einer gemeinsamen virtuellen Bibliothek zugänglich zu machen. Das Parallelprojekt in Österreich ist manuscripta.at https://manuscripta.at/digitalisate.php (07.06.2020). In Deutschland gibt es m.W. keine vergleichbare Initiative. Hier bieten die einzelnen Forschungsinstitutionen ihre Bestände digital an, siehe etwa die digitalen Sammlungen der Staatsbibliothek Berlin https://digital.staatsbibliothek-berlin.de/ (08.06.2020) oder des Münchner Digitalisierungszentrums https://www.digitale-sammlungen.de/ (08.06.2020). Jedoch führt das Projekt »Manuscripta Mediaevalia« http://www.manuscripta-mediaevalia.de (08.06.2020) zahlreiche digitalisierte Handschriftenkataloge zusammen, was die Auffindung sehr erleichtert. 
Mehr noch als bei digitalisierten Texteditionen, die eher die Anwendung der klassischen Methoden erleichtern, kann hier ein Einfluss des technischen Fortschritts auf die Entwicklung des Fachs selbst beobachtet werden. Es ist sicherlich kein Zufall, dass mit den Möglichkeiten der Digitalisierung seit den 1980er Jahren der sogenannte »material turn « einhergeht, also eine verstärkte Hinwendung zu den historischen Objekten und ihrer Materialität. In diesem Zusammenhang werden Handschriften nicht mehr primär oder ausschließlich als Träger von Texten, sondern in ihrem eigenen, materialen Quellenwert angesehen und erforscht. Welche Auswirkungen das auf die Entwicklung von neuen Forschungsfragen und -methoden hat, zeigen einerseits der interdisziplinäre Sonderforschungsbereich »Materiale Textkulturen « in Heidelberg (https://www.materiale-textkulturen.de/ [08.06.2020]) und andererseits das Projekt e-codicology der TU Darmstadt, das sich die Entwicklung neuer Algorithmen zur Erfassung mikro- und makrostruktureller Gestaltungsmerkmale mittelalterlicher Handschriften zum Ziel gesetzt hat, welche vor allem sozial-, wirtschafts- und kulturgeschichtliche Erkenntnisse über die Trägerkreise erlauben. Für die Kirchengeschichte, die ganz wesentlich auch eine texthermeneutische Wissenschaft ist, wird die Zukunft wohl in einer Verknüpfung von Methoden des »distant reading « mit dem klassischen »close reading « der Quelleninterpretation liegen. In diese Richtung weisen jüngste Forschungsprojekte wie etwa das vom ERC finanzierte Projekt »Paratexte der Bibel « in München (http://paratexbib.eu/) oder das SNF-Sinergia-Projekt »Reading Josephus in the Latin Middle Ages « in Bern (https://legejosephum.ch/en [beide 08.06.2020]). Hier wird aufgrund der Materialstudien an den Handschriften nach Bezügen zwischen Textinhalten und Gestaltung der Objekte gefragt, nach Benutzerspuren in Marginalien, nach dem Zusammenspiel von Text und Illustration. Damit gewinnt das einzelne Objekt an Bedeutung gegenüber dem aus (mehreren Textzeugen erstellten) kritischen Text.

Die hochwertige Digitalisierung von Handschriften hat allerdings nicht nur das Interesse am materialen Objekt gesteigert und die Zugänglichkeit enorm erleichtert, sie bringt paradoxerweise auch eine neue Entzogenheit der Quellen mit sich. Vielerorts wird in Handschriftenabteilungen die Aushändigung der Codices unter Hinweis auf die Digitalisate erschwert.

\section{c) Digitale Editionen}

Eine naheliegende, aber zugleich paradoxe Konsequenz aus dieser Entwicklung ist, dass der vereinfachte Zugang zu Texteditionen und Handschriften in Digitalisaten die textkritische Arbeit nicht nur erleichtert, sondern ihr Ideal eines »Urtextes « zugleich in Frage stellt.

Wie sinnvoll ist es, den wahrscheinlichsten ursprünglichen Wortlaut eines Textes aus einer Vielzahl von Textzeugen zu (re)konstruieren, wenn erstens alle Welt einen direkten Zugang zu immer mehr dieser Textzeugen hat und zweitens im Zuge der »material studies« deutlich wird, wie hoch der Quellenwert dieser 
Objekte gegenüber einem Text ist, der in dem kritisch (re)konstruierten Wortlaut womöglich nie von irgendeinem Menschen geschrieben oder gelesen wurde? Vor diesen Fragen, für die es keine einfachen Antworten gibt, stehen Forschende, die genuin digitale Editionen erstellen. Sollen sie das Ideal der kritischen Edition bewahren oder sich auf die Transkription einzelner Handschriften beschränken und möglichst optimale technische Lösungen zu deren Vergleich durch die Nutzer suchen?

Die (zumindest im deutschsprachigen Raum) erste kritisch-digitale Edition eines für die ältere Kirchengeschichte relevanten Quellentextes besorgte der Philologe F. Fischer für Wilhelm von Auxerre, »Summa de officiis ecclesiasticis « (http:// guillelmus.uni-koeln.de/tcrit/tcrit_prologus [08.06.2020]). Die 2007 erstellte und 2013 gründlich revidierte Webseite bietet einen aus 15 Handschriften kritisch erstellten Text mit integrierten text- und quellenkritischem Apparaten sowie Faksimiles bzw. Digitalisate der einzelnen Handschriften. Ob sich Kompromisse dieser Art bewähren werden, wird die Zukunft der digitalen Editionsforschung zeigen (siehe dazu die Ausführungen von S. Chronopoulos/F.K. /Maier/A. Novokhatko (https://doi.org/10.11588/propylaeum.563 [08.06.2020]).

\section{Fallbeispiel: Augustinus" "Confessiones" digital}

Es ist ein seltener Luxus, wenn, wie im Fall des Corpus Thomisticum, eine Webseite (bzw. die dahinter stehende wissenschaftliche Community) alle verfügbaren Hilfsmittel für ein kirchenhistorisches Forschungsfeld nach wissenschaftlichen Qualitätskriterien prüft und (nur!) die für gut befundenen digital zur Verfügung stellt. Im Normalfall sind Forschende auf Suchmaschinen angewiesen und unternehmen eine allenfalls graduell systematische Recherche, die stark von Zufällen (bzw. von Algorithmen) bestimmt ist, ohne dass die Suchenden die Auswirkungen dieser Tatsache abschätzen und für ihre Forschung kalkulieren könnten oder auch nur im Blick hätten.

Augustinus gehört (neben Thomas von Aquin) zu den wenigen Autoren der Kirchengeschichte, denen eine eigene Meta-Seite gewidmet ist. Die Webseite des Zentrums für Augustinus-Forschung an der Universität Würzburg (www.augustinus.de) bietet einen hilfreichen Einstieg in den digitalen Augustinus, auch wenn sie sich in einer etwas eigenwilligen Mischung aus einführenden Informationen zu Leben und Werk des Augustinus, Links zu Editionen und Übersetzungen, Links zu Datenbanken und Bibliothekskatalogen, Veranstaltungshinweisen des Zentrums sowie erbaulichen Augustinus-Zitaten und Bildern ohne Bildnachweis präsentiert.

Besonders nützlich für das Studium des Originaltextes sind zwei Links: Die kritische Werkausgabe des Corpus Augustinianum Gissense online https://cag-online.net/users/login (08.06.2020) bietet den maßgeblichen lateinischen Text mit kritischen Apparaten. Für das Verständnis zentraler Begriffe und Realia ist die Online-Ausgabe des Augustinus-Lexikons ein hervorragendes Hilfsmittel. Beide Datenbanken basieren auf vorgängigen Printversionen und wurden hervorragend digital aufbereitet, der Zugang muss allerdings kostenpflichtig in der Digitalen Bib- 
liothek des Schwabe-Verlags erworben werden (https://al-online.ch [05.07.2020]). So mag für diejenigen, die sich auf www.augustinus.de verlassen, der Eindruck entstehen, es gäbe keinen frei zugänglichen, kritischen lateinischen Text der Confessiones im Internet. Aber dieser Eindruck ist irreführend. Vielmehr bietet die oben genannte Bibliotheca Augustana unter http://www. hs-augsburg.de/ harsch/Chronologia/Lspost05/Augustinus/aug_c000.html (08.06.2020) Links zu zwei kritischen Ausgaben des lateinischen Textes (CSEL 32, ed. Knöll, Leipzig 1896 und ed. Verheijen, Turnhout 1981), beide allerdings ohne die text- und quellenkritischen Apparate der Printversionen.

Für die inhaltliche Erschließung der Confessiones am hilfreichsten ist die (ebenfalls auf der Bibliotheca Augustana verlinkte) digitale Aufbereitung des Buches »Augustine: Confessions, a text and commentary « von J.J. O'Donnell (http:// www.stoa.org/hippo/index.html [08.06.2020]). Text und Kommentare wurden in SGML codiert, Einleitungen, Kommentare, Fußnoten und Referenzen auf biblische und andere klassische Texte sowie auf Sekundärliteratur direkt verlinkt. Zurück zum Zentrum für Augustinus-Forschung und der Suche nach Übersetzungen der Confessiones in moderne Sprachen: Unter dem Reiter »Online-Übersetzungen Augustinus « werden ausschließlich frei zugängliche Seiten aufgelistet, im Fall der Confessiones drei deutsche und drei englische sowie je eine französische, spanische und italienische.

Wie so häufig, findet man auch in diesem Fall im Internet nur Übersetzungen älteren Datums, die gemeinfrei sind. Unter den drei deutschen Seiten bieten zwei die Übersetzung von Otto Lachmann aus dem Jahr 1888: zum einen als von der Universität Freiburg i.B. zur Verfügung gestelltes PDF (https://www.ub.uni-freiburg.de/fileadmin/ub/referate/04/augustinus/lachmann. pdf [08.06.2020]), zum anderen als Intratext mit Wortlisten und Konkordanzen (http://www. intratext.com/IXT/DEU0016/_INDEX.HTM [08.06.2020]).

Als nützlichstes Tool für die Texterschließung der Confessiones erweist sich aber die - wiederum nicht über das Zentrum für Augustinus-Forschung verlinkte - digitale Ausgabe der Bibliothek der Kirchenväter, welche allerdings mit der Übersetzung von Alfred Hofmann ebenfalls einen über einhundert Jahre alten deutschen Text bietet. Das in Fribourg $(\mathrm{CH})$ koordinierte Projekt bot in seiner ersten Auflage seit 2003 (http://www.unifr.ch/bkv/ [diese Seite ist noch aktiv, wird aber nicht mehr aktualisiert, sondern verweist auf die neue Version]) die zwischen 1831 und 1938 im Münchner Kösel-Verlag erschienenen 200 Bände der BKV in durchsuchbaren rtf- und PDF-Formaten. Die zweite Auflage (https:// bkv.unifr.ch/ [08.06.2020]) wird seit 2018 mit digitalen Tools »aufgerüstet «. So findet man jetzt auch Textversionen in der Originalsprache und kann im Fall der Confessiones den lateinischen Text und die deutsche Übersetzung synoptisch nebeneinanderstellen. Während die deutschen Übersetzungen trotz der etwas altertümlichen Sprachgestalt philologisch zumeist sehr gut (und von den Editoren teilweise leicht überarbeitet) sind, ist im Hinblick auf die Vorworte, Einleitungen und Kommentare Vorsicht geboten, da sie den Forschungsstand und die akademischen Ansichten von Gelehrten des 19. Jh. repräsentieren. 
Soweit zum bloßen Text dieses vielleicht einflussreichsten Werks der christlichen Antike. Wer auf der Suche nach Handschriften der Confessiones ist, wird auf der Webseite des Zentrums für Augutinus-Forschung nicht fündig. Und da es ein lateinisches Äquivalent zu Pinakes, der oben erwähnten Datenbank für griechische Handschriften weltweit, bislang nicht gibt, muss man einzelne Bibliotheken oder übergreifende Datenbanken aufsuchen.

Zusammenfassend lässt sich sagen, dass die Metaseite des Zentrums für AugustinusForschung ihren Nutzern einen Zugang zum lateinischen und deutschen Text der Confessiones ermöglicht. Wer die Seite allerdings für akademische Zwecke nutzen will, ist auf die kostenpflichtigen Links angewiesen und muss zahlen. Die mittelalterlichen Manuskripte werden leider gar nicht erschlossen. Auch eine frei zugängliche allgemeine Einführung zum Werk sucht man vergebens.

Die üblichen Literatursuchmaschinen und -datenbanken bieten bei einer Recherche mit dem Stichwort »Confessiones « natürlich zahlreiche Treffer auf digitalisierte Forschungsliteratur, die allerdings nur schwer überschaubar und häufig ebenfalls nicht frei zugänglich ist. Die für Viele (nicht nur für Laien, sondern - seien wir ehrlich! - auch für Akademiker) naheliegende Annahme ist, dass man eine allgemeine Einführung mit nützlichen Links am schnellsten bei Wikipedia findet. Werfen wir also einen kurzen Blick auf den entsprechenden Eintrag in Wikipedia. Im ersten Satz werden die Confessiones als "autobiographische Betrachtungen « gekennzeichnet, ohne dass der Forschungsdiskurs darüber, ob das Werk als Autobiographie konzipiert war und so rezipiert werden kann, erwähnt würde. Dennoch hat dieser Diskurs im Artikel Spuren hinterlassen, denn auf das Kapitel »Inhalt (welches irritierenderweise zunächst über »Allgemeines « und dann im Einzelnen über die Bücher 8-13 informiert), folgen Ausführungen zu »Programmatik des Titels«, »Gattung « und »Rezeption«. Damit sind wichtige Themen der Augustinus-Forschung angesprochen. Problematisch ist, dass die in diesen Kapiteln vorfindlichen Angaben zu Inhalt, Charakter, Intention und Quellenwert des Werkes in Spannung, ja in Widerspruch zueinander stehen. So liest man unter »Allgemeines «, die Confessiones enthielten »zahlreiche Informationen über den spätrömischen Alltag «, woraus man einen hohen sozialgeschichtlichen Quellenwert ableiten möchte. Die dann aufgeführten »Grundlinien« des Werkes sind nicht unzutreffend, illustrieren aber den sozialgeschichtlichen Wert gerade nicht, sondern fokussieren auf theologische und anthropologische Aussagen. Die Inhaltsangaben » $\mathrm{Zu}$ einzelnen Büchern« setzen erst bei Buch 8 ein und thematisieren ausschließlich philosophisch-theologische Themen, unter Einstreuung teils nicht belegter lateinischer Zitate. Die Aussage, es finde sich »weniges, was allzu persönlich und deshalb nicht übertragbar wäre«, steht in Spannung zur Definition als Autobiographie im ersten Satz des Artikels. Die Abschnitte zum Werktitel und zur literarischen Gattung geben einen recht instruktiven Einblick in die von der Forschung diskutierten Fragen, stehen aber wiederum in Spannung bzw. Widerspruch zu vorigen Abschnitten und sind auch selbst nicht ganz fehlerfrei. (Jedenfalls hätte der bekennende Platoniker Augustinus wohl Einwände gegen die Zuweisung des »Protreptikos« an die Sophisten gehabt.) 
Die Auflistung deutscher Übersetzungen, die Einzelnachweise, Literaturhinweise und Weblinks, welche zu jedem Wikipedia-Artikel gehören, sind recht gut ausgewählt und weiterführend, können bei einem Thema wie diesem aber eine eigene Recherche in wissenschaftlichen Datenbanken selbstverständlich nicht ersetzen. Leider fehlt der Link auf die Bibliothek der Kirchenväter (https://bkv.unifr.ch/), welche unter den online verfügbaren Textausgaben aufgrund der Möglichkeit zur zweisprachigen Synopse die nützlichste ist (s.o.). Für das beigegebene Bild, eine Darstellung der Taufe des Augustinus des Renaissancekünstlers Benozzo Gozzoli, findet sich weder eine Erklärung noch ein vollständiger Bildnachweis.

Die Versionsgeschichte - ein Ausweis der Transparenz von Wikipedia - ist in dieser Hinsicht sehr aufschlussreich: Seit der Einrichtung des Stichworts durch einen Unbekannten am 23.02.2003 wurde er in 189 redaktionellen Operationen bearbeitet, die aktuelle Version (zuletzt eingesehen am 20.04.2020) stammt vom 23.03.2020 und hat eine Größe von 19763 Bytes. Unter den mehr als 100 Bearbeitern finden sich, ausweislich der Benutzerprofile, ein 1954 geborener »Familienvater, Informatiker und Unternehmer «; ein anonymer » Wissensdurstiger «, eine methodistische Schweizerin mit Jahrgang 1951, ein promovierter Philosoph und Ingenieur, ein katholischer Priester aus Tirol, ein »Victor Eremita (eines der Pseudonyme des dänischen Philosophen Søren Kierkegaard, dessen Portrait auch auf dem Benutzerprofil erscheint) sowie eine »Morgenröte«. Die umfangreichsten Ergänzungen haben 2008 ein Benutzer mit dem Namen Ca\$e und im Frühjahr 2012 in mehreren Schritten ein 1983 geborener evangelischer Theologe vorgenommen. Auf ersteren gehen die lateinischen Zitate und philologischen Bemerkungen, auf letzteren die Informationen zu philosophisch-theologischen Themen zurück.

Insgesamt enthält der Wikipedia-Artikel zwar viele Informationen und Hinweise auf Forschungsergebnisse und -diskurse. Diese stehen jedoch unsystematisch und teilweise einander widersprechend nebeneinander. Die eigentümliche Mischung aus brauchbaren Informationen und fehlender Stringenz ist typisch für Wikipedia und hängt wohl mit der Redaktionspolitik zusammen, von Streichungen fremder Einträge möglichst abzusehen. Es bedarf einer hohen Kompetenz und viel Vorwissens, um Erkenntnis daraus zu ziehen. So verhält es sich mit dem Wikipedia-Artikel wie mit seinem Gegenstand, den Confessiones, selbst: Sie mit Gewinn zu lesen, ist für das Studium der älteren Kirchengeschichte ebenso naheliegend wie anspruchsvoll. Als Einführung in die Confessiones erweist sich das Kapitel von P. Frederiksen im »Augustin Handbuch « (hg.v. V.H. Drecoll, Tübingen 2007, e-book 2014, 294-309), 2007 in Printversion und 2014 als e-book PDF erschienen, immer noch als die bessere Alternative sowohl zu Wikipedia als auch zum Internetauftritt des Zentrums für Augustinus-Forschung.

\section{Fazit: Das Internet als Ernstfall der Heuristik}

In mindestens drei Aspekten zeigen die hier zusammengestellten Beobachtungen, dass und in welcher Weise sich altbekannte Herausforderungen für die kirchengeschichtliche Forschung im Internet wiederholen und teilweise auch verschärfen: Erstens hängen Anzahl und Art der verfügbaren Quellen ebenso von Steuerung 
wie von Zufällen der Überlieferung ab. Für die Qualität der Erkenntnisse ist es wichtig, sich diese Zusammenhänge zu verdeutlichen und sie zu reflektieren. $\mathrm{Zu}$ nächst reproduziert das Internet, indem es (jedenfalls gegenwärtig noch) vor allem nachträglich digitalisierte Printmedien präsentiert, die vorhandenen Bestände und potenziert damit automatisch auch die bisher wirkmächtigen Auswahlmechanismen, etwa bei Quellenreihen patristischer Literatur. Daher ist zu fragen, ob die Behauptung, das Internet trage zur Ent-Kanonisierung und Demokratisierung der Wissenschaft bei, im Blick auf die Kirchengeschichte wirklich zutreffend ist. Verschärfend wirkt sich der Umstand aus, dass wegen des Urheberschutzes vornehmlich ältere Printmedien frei zugänglich sind. Daraus resultiert der paradoxe Zustand, dass ausgerechnet das modernste Medium häufig die ältesten Editionen, Übersetzungen und Forschungsbeiträge präsentiert. Da dies bisweilen in recht modernem Design geschieht, darf man sich vom Erscheinungsbild nicht täuschen lassen. Ob genuin digital erzeugte Editionen in Zukunft zu einer Ent-Kanonisierung der Kirchengeschichte oder doch eher zu einem Revival der »Monumentalgeschichte « im Internet beitragen werden, muss sich erst noch weisen.

Zweitens stellt sich das Problem der zugleich notwendigen wie unmöglichen Kontextualisierung der aufgefundenen Quellen. Hier ergeben sich erstaunliche Analogien zwischen der Suche nach historischen Quellen und Recherchen im Internet. Beide Felder sind prinzipiell unüberschaubar, bei vielen Entdeckungen ist Zufall im Spiel. Allerdings sind die Gründe dafür verschieden: Im Blick auf die Vergangenheit haben wir es mit einem Mangel, im Internet eher mit einer Überfülle an vorhandenen Informationen zu tun. Die Konsequenz bleibt jedoch dieselbe: Es wäre in beiden Fällen wissenschaftlich unredlich, zufällige Funde und daraus resultierende Erkenntnisse als repräsentative Punktlandungen zu verkaufen. Es ist und bleibt also, auch und gerade für internetbasierte Forschung, ein wissenschaftlicher Qualitätsausweis, das eigene Unwissen und die möglichen Auswirkungen auf die Forschungsergebnisse zu reflektieren und möglichst präzise zu beschreiben. Drittens werden Bilder und andere nicht-textliche Quellen sowohl in der analogen als auch in der digitalen Präsentation kirchengeschichtlicher Forschung viel zu oft als bloße Illustrationen und viel zu selten als Quellen eigenen Werts verwendet. Dies hat vor allem das Beispiel der Webseiten zu Augustinus gezeigt, die kommentar- und nachweislos mit neuzeitlichen Gemälden geschmückt sind. Auch hier verlängert und verschärft das Internet eine lange Tradition der Vernachlässigung und unangemessenen Verwendung von Bildquellen in der Kirchengeschichte. Sie kann und sollte von den »Digital Humanities « und deren Aufbereitung und Präsentation digitaler Sammlungen von Handschriften und anderen Objekten lernen. Ein erstes hilfreiches Tool für die Suche nach ikonographischen und archäologischen Quellen ist das kostenpflichtige Bildarchiv Prometheus (https:// www.prometheus-bildarchiv.de/ [08.06.2020]). Dabei handelt es sich um einen sogenannten »Datenbankbroker «, in dem momentan 103 heterogene Datenbanken verschiedener Institutionen zusammengeführt sind.

Insgesamt gilt im Umgang mit dem Internet, was in der kirchengeschichtlichen 
Forschung schon immer galt: Die Forschungsfragen müssen so gestellt werden, dass sie mit dem verfügbaren Quellenbestand bearbeitet werden können, ohne ihn einfach unkritisch zu referieren. Die Verschärfung besteht darin, dass der im Internet verfügbare Quellenbestand und seine Präsentationsformen rasant und stetig wachsen. Wer die »Bergmannskunst « der historischen Heuristik beherrscht, dürfte daher für die Abwägung von Nutzen und Nachteil des Internets in der Kirchengeschichte (nur, aber immerhin!) einigermaßen gut gerüstet sein.

\section{Netzwerke, historisch und digital Digital Humanities und die Mittlere und Neue Kirchengeschichte \\ Ueli Zahnd}

Brepolis, the home of Brepols' online publications, Brepols Turnhout, online: http://www.brepolis. net/ (12.06.2020). - correspSearch. Briefeditionen durchsuchen und vernetzen, Berlin-Brandenburgische Akademie der Wissenschaften, online: https://correspsearch.net (12.06.2020). - HyperImage, bitGilde IT Solutions Berlin, http://hyperimage.ws/ (12.06.2020). - International Mission Photography Archive = IMPA, University of Southern California Los Angeles(California), online: http:// digitallibrary.usc.edu/cdm/landingpage/collection/p15799coll123 (12.06.2020). - Post-Reformation Digital Library, hg.v. David S. Sytsma, Junius Institute for Digital Reformation Research Grand Rapids (Michigan), online: http://www.prdl.org/ (12.06.2020). - Religiöse Friedenswabrung und Friedensstiftung in Europa, hg.v. Irene Dingel, Leibniz-Institut für Europäische Geschichte Mainz und Technische Universität Darmstadt, online: http://www.religionsfrieden.de/ (12.06.2020). - The Scholastic Commentaries and Texts Archive, hg.v. Jeffrey C. Witt, Loyola University of Maryland Baltimore (Maryland), online: https://scta.info/ (12.06.2020). - Theologenbriefwechsel im Südwesten des Reichs in der Frühen Neuzeit (1550-1620), hg.v. Christoph Strohm, Heidelberger Akademie der Wissenschaften, online: http://www.hadw-bw.de/thbw.html (12.06.2020).

Weitere Literatur

Christian Bizer/Tom Heath/Tim Berners-Lee, Linked Data - The Story So Far: International Journal on Semantic Web and Information Systems 5 (2009) 1-22, DOI: 10.4018/jswis.2009081901 (12.06.2020). - Fabio Ciotti/Francesca Tomasi, Formal Ontologies, Linked Data, and TEI Semantics: Journal of the Text Encoding Initiative 9 (2016/17) 22 Abs., DOI: 10.4000/jtei.1480 (12.06.2020). - Stefan Dumont, correspSearch: Connecting Scholarly Editions of Letters: Journal of the Text Encoding Initiative 10 (2016) 28 Abs., DOI: 10.4000/ jtei.1742 (12.06.2020). - Elena Pierazzo, Digital Scholarly Editing. Theories, Models and Methods, Ruotledge Taylor and Francis London 2015, 236 S. - Patrick Sable, Mal wieder und immer noch: Digitzed vs. Digital (27.11.2012), online: https://dhd-blog.org/?p=1122 (12.06.2020). - Joris van Zundert, Barely Beyond the Book?: Matthew J. Driscoll/Elena Pierazzo (Hg.), Digital Scholarly Editing. Theories and Practices, OpenBook Cambridge 2016, 83-106, online: https://books.openedition.org/obp/3402 (12.06.2020). - Jeffrey C. Witt, Digital Scholarly Editions and API Consuming Applications: Roman Bleier/Martina Bürgermeister/Helmut W. Klug/Frederike Neuber/Gerlinde Schneider (Hg.), Digital Scholarly Editions as Interfaces (Schriften des Instituts für Dokumentologie und Editorik 12), Books on Demand Norderstedt 2018, 219-247.

114 Verkündigung und Forschung 65. Jg., Heft 2, S. 114-123, ISSN 0342-2410, @ 2020 Gütersloher Verlagshaus, Gütersloh, in der Verlagsgruppe Random House GmbH, Neumarkter Str. 28, 81673 München 